13

\title{
Высокоэффективный гиротрон с многоступенчатой рекуперацией остаточной энергии электронов
}

\author{
(C) О.И. Лукша, П.А. Трофримов \\ Санкт-Петербургский политехнический университет Петра Великого, \\ 195251 Санкт-Петербург, Россия \\ e-mail: louksha@rphf.spbstu.ru
}

Поступило в Редакцию 30 апреля 2019 г.

В окончательной редакции 30 апреля 2019 г.

Принято к публикации 21 мая 2019 г.

Представлены результаты комплексного моделирования физических процессов в гиротроне средней мощности 4-mm диапазона длин волн излучения. Реализованы методы повышения качества винтового электронного потока и электронного КПД гиротрона, основанные на оптимизации распределения электрического поля в прикатодной области. Разработана конструкция коллектора с 4-х ступенчатой рекуперацией остаточной энергии пучка, основанная на методе пространственной сепарации электронов в скрещенных азимутальном магнитном и аксиальном электрическом полях. За счет повышения качества электронного пучка и эффективной рекуперации энергии в коллекторной области достигнуто значение полного КПД гиротрона, равное $71.8 \%$.

Ключевые слова: СВЧ электроника, гиротрон, электронный поток, рекуперация энергии.

DOI: 10.21883/JTF.2019.12.48501.179-19

\section{Введение}

В настоящее время гиротроны занимают лидирующее положение среди источников мощного СВЧ излучения в миллиметровом и субмиллиметровом диапазонах длин волн. Гиротроны мегаваттного уровня мощности, работающие в длинноимпульсном и квазинепрерывном режимах, востребованы для нагрева плотной плазмы и управления током в установках управляемого термоядерного синтеза. Эти приборы применяются также при обработке материалов, в спектроскопии высокого разрешения, для диагностики различных сред и в других приложениях.

Эффективность взаимодействия винтового электронного потока (ВЭП) с высокочастотным полем в резонаторе гиротронов относительно невысока. Как правило, электронный КПД мощных гиротронов не превышает $30-35 \%$ [1-3]. Повышения эффективности можно добиться в результате оптимизации системы формирования ВЭП, нацеленной на создание пучка высокого качества с большим питч-фактором, малым скоростным и энергетическим разбросами, требуемой пространственной структурой [4]. Величиной питч-фактора $\alpha=v_{\perp} / v_{\|}$ $\left(v_{\perp}\right.$ и $v_{\|}$- поперечная и продольная компоненты скорости электрона) принято характеризовать долю сосредоточенной в поперечном движении электронов энергии, из которой „черпается“ энергия выходного СВЧ излучения в гирорезонансных приборах.

В качестве примера подобной оптимизации можно привести гиротроны мегаваттного уровня мощности с частотами 110 и $140 \mathrm{GHz}$ и электронным КПД более $40 \%$, изготовленные в ИПФ РАН [2,5]. Экспериментальные исследования, направленные на повыше- ния качества ВЭП, были также проведены в СПбПУ с помощью импульсного гиротрона с рабочей частотой $74.2 \mathrm{GHz}$ и выходной мощностью примерно $100 \mathrm{~kW}$ [6-11]. В результате этих исследований электронный КПД гиротрона был повышен до 46\% за счет оптимизации распределений электрического и магнитного полей в области формирования ВЭП, а также увеличения однородности термоэлектронной эмиссии с катода [11].

Повышение полного КПД гиротронов может быть достигнуто в результате рекуперации - возвращения в электрическую сеть части энергии электронного потока, не израсходованной при его взаимодействии с высокочастотным полем. Практически все современные мощные гиротроны оснащены системами одноступенчатой рекуперации (single-stage depressed collector), в которых торможение электронов происходит в зазоре между корпусом лампы и изолированным от него коллектором. Такие системы позволяют значительно повысить полный КПД гиротрона (до 1.5 раз по сравнению с электронным КПД при отсутствии рекуперации $[1,2,12])$. Принципиально важно для мощных длинноимпульсных и квазинепрерывных гиротронов, что при рекуперации остаточной энергии электронов снижается также тепловая нагрузка на коллектор.

Дальнейшее увеличение полного КПД возможно при использовании систем многоступенчатой рекуперации, которые предполагают разделение в пространстве фракций пучка с разными энергиями и осаждение этих фракций на секции коллектора под различными потенциалами (например, [13-17]). Однако на практике такие системы, насколько нам известно, еще не были реализованы в приборах гиротронного типа. Это связано 
Таблица 1. Основные геометрические параметры гиротрона и характеристики его рабочего режима

\begin{tabular}{l|l}
\hline \multicolumn{1}{c|}{ Параметр } & \multicolumn{1}{c}{ Значение } \\
\hline Ускоряющее напряжение & $U_{0}=30 \mathrm{kV}$ \\
Ток пучка & $I_{b}=10 \mathrm{~A}$ \\
Индукция магнитного поля в области & \\
резонатора & $B_{0}=2.75 \mathrm{~T}$ \\
Индукция магнитного поля у катода & $B_{c}=0.152 \mathrm{~T}$ \\
Рабочая мода & $\mathrm{TE}_{12,3}$ \\
Рабочая частота & $f_{0}=74.2 \mathrm{GHz}$ \\
Радиус резонатора & $R_{0}=14.45 \mathrm{~mm}$ \\
Средний радиус эмитирующего пояска & \\
катода & $R_{c}=35 \mathrm{~mm}$
\end{tabular}

со спецификой электронных траекторий в коллекторной области этих приборов, имеющимся разбросом электронов по компонентам скорости и координатам, наличием относительно сильного магнитного поля. Новые возможности для сепарации электронов с разной энергией открываются при использовании подхода, основанного на их дрейфе в скрещенных электрическом и магнитном полях [18-20]. На основе такого подхода в СПбПУ был разработан многоступенчатый рекуператор, в котором дрейф электронов осуществляется в азимутальном магнитном и аксиальном электрическом полях $[21,22]$.

В настоящей работе представлены результаты комплексного моделирования процессов в гиротроне СПбПУ, включающих формирование ВЭП в электроннооптической системе (ЭОС), взаимодействие электронов с высокочастотным полем и транспортировку пучка в коллекторной области. Данное исследование имело целью достижение рекордных значений полного КПД гиротрона как за счет формирования высококачественного ВЭП, так и за счет эффективной рекуперации остаточной энергии в многоступенчатой коллекторной системе. Расчеты были выполнены с использованием программного пакета 3D-моделирования CST Studio Suite [23]. На разных этапах моделирования было произведено сопоставление полученных данных с результатами экспериментов, выполненных в данном гиротроне.

\section{1. Траекторный анализ ВЭП в электронно-оптической системе гиротрона}

Формирование ВЭП в гиротроне СПбПУ осуществляется с помощью трехэлектродной магнетронноинжекторной пушки (МИП) (рис. 1). В состав катодного блока пушки входит управляющий электрод, изменением потенциала которого можно изменять средний питч-фактор и скоростной разброс электронов $[9,24]$. Основные параметры гиротрона приведены в табл. 1 . Указанные в этой таблице значения $U_{0}, I_{b}, B_{0}$ и $B_{c}$ соответствуют расчетному рабочему режиму гиротрона со средним питч-фактором, равным примерно 1.3. В описанных ниже расчетах, как и в экспериментах, значения этих величин варьировались, что позволяло изменять характеристики пучка на входе в резонатор и генерируемую СВЧ мощность в пределах рабочей моды $\mathrm{TE}_{12.3}$. Самосогласованные электронные траектории рассчитывались с помощью вычислителя Tracking Solver. Были исследованы два режима термоэлектронной эмиссии с катода. В первом режиме (однородный катод) эмиссия с одинаковой плотностью тока осуществлялась со всего эмитирующего пояска. Поток в этом случае состоял из 3600 электронных траекторий - трубок тока. Во втором режиме (секционированный катод, 2200 траекторий) отсутствовала эмиссия с двух азимутальных секторов по $70^{\circ}$ каждый, положение которых соответствовало положению „связок“ тороидального коллекторного соленоида (см. ниже разд. 3). При моделировании эмиссии учитывался разброс электронов по начальным тепловым скоростям. В обоих режимах полный ток ВЭП составлял 10 А. Распределение магнитного поля при заданной геометрии катушек определялось с помощью вычислителя Magnetostatic Solver и затем экспортировалось в текущий проект. Расчетная модель при траекторном анализе разбивалась примерно на $80 \cdot 10^{6}$ ячеек.

Изменением величины магнитной компрессии $B_{0} / B_{c}$ можно регулировать средний радиус полого ВЭП в резонаторе и, как следствие, выходную СВЧ мощность. В данных расчетах величина $B_{0} / B_{c}$ изменялась за счет варьирования числа витков катодной катушки [6]. Максимальные значения выходной мощности были получены при $B_{0} / B_{c}=17.01$, что соответствует 26 виткам этой катушки. При заданном $B_{0} / B_{c}$ варьированием значений потенциалов анода $U_{a}$ и управляющего электрода $U_{\text {cont }}$ выбирался режим с минимальным скоростным разбросом $\delta v_{\perp}$ и средним питч-фактором $\alpha$ в диапазоне 1.5-1.6 (потенциалы катода $U_{c}=-30 \mathrm{kV}$ и корпуса прибора $U_{\text {body }}=0$ при этом не менялись). Здесь и далее в разд. 1 значения параметров ВЭП относятся к центральной плоскости резонатора $z=260.5 \mathrm{~mm}$ (рис. 1). Отметим, что ранее в экспериментах максимальный КПД гиротрона был достигнут при расчетном значении $\alpha$, равным примерно 1.6 [11].

В режиме с $B_{0} / B_{c}=17.01, U_{a}=8.4 \mathrm{kV}$ и $U_{\text {cont }}=$ $=-44.5 \mathrm{kV}$ были получены следующие значения параметров ВЭП: $\alpha=1.57, \delta v_{\perp}=2.8 \%$ для однородного катода и $\alpha=1.52, \delta v_{\perp}=3.4 \%$ для секционированного катода. Величина $\delta v_{\perp}$ определялась как среднеквадратичное отклонение от среднего значения поперечной скорости. Распределение частиц в плоскости поперечного сечения ВЭП для обоих катодов показано на рис. 2. Неоднородная эмиссия с катода является одним из факторов, ухудшающих качество ВЭП (например, [24]). Поэтому при переходе от однородного к секционированному катоду увеличивается разброс электронов по скоростям и по энергиям, а также возникает дополнительное смещение электронов по радиусу изза действия скрещенных азимутального электрического 


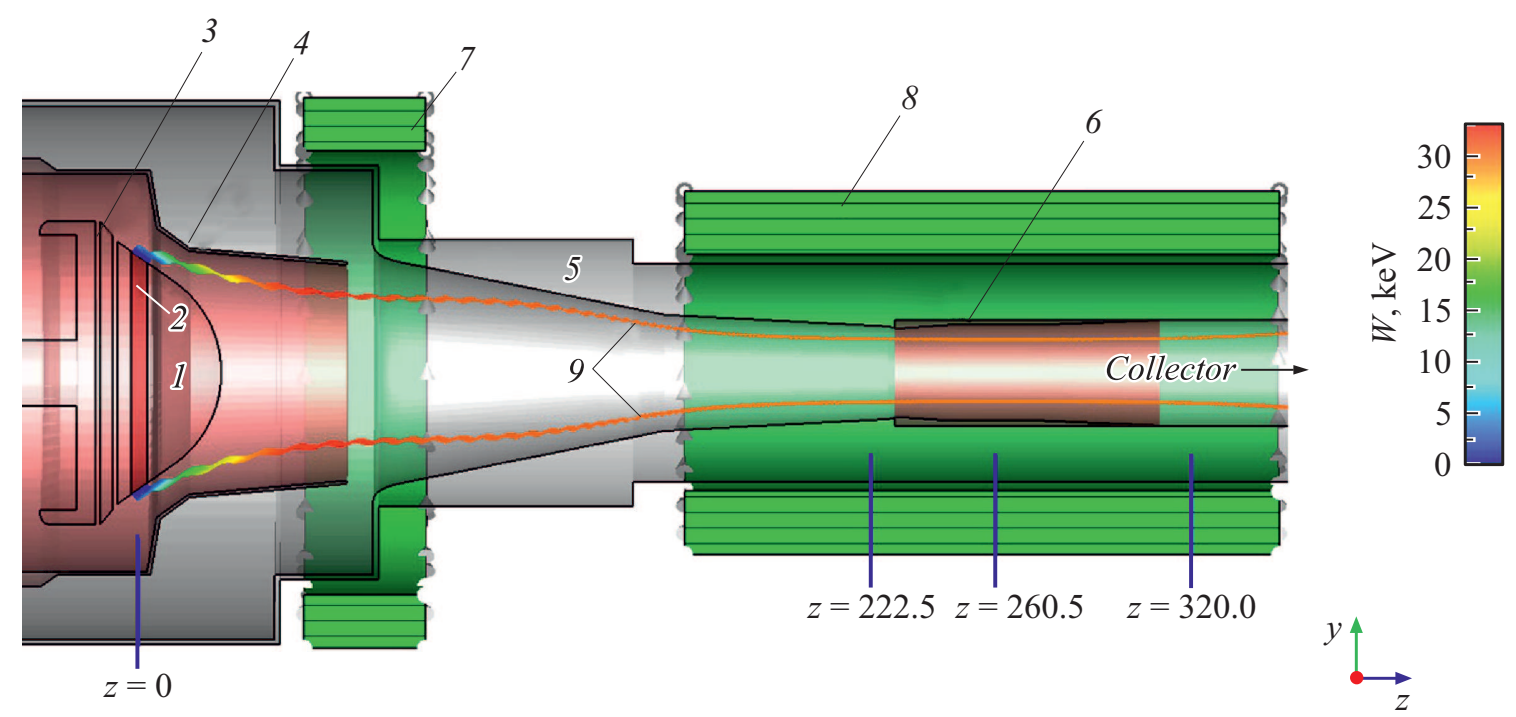

Рис. 1. Схематическое изображение модели гиротрона: 1 - катод, $2-$ эмитирующий поясок, $3-$ управляющий электрод, $4-$ анод, 5 - корпус прибора, $6-$ резонатор, 7 - катодная катушка, $8-$ основной соленоид, $9-$ траектории электронов (цвет траекторий соответствует энергии частиц $W$ ).
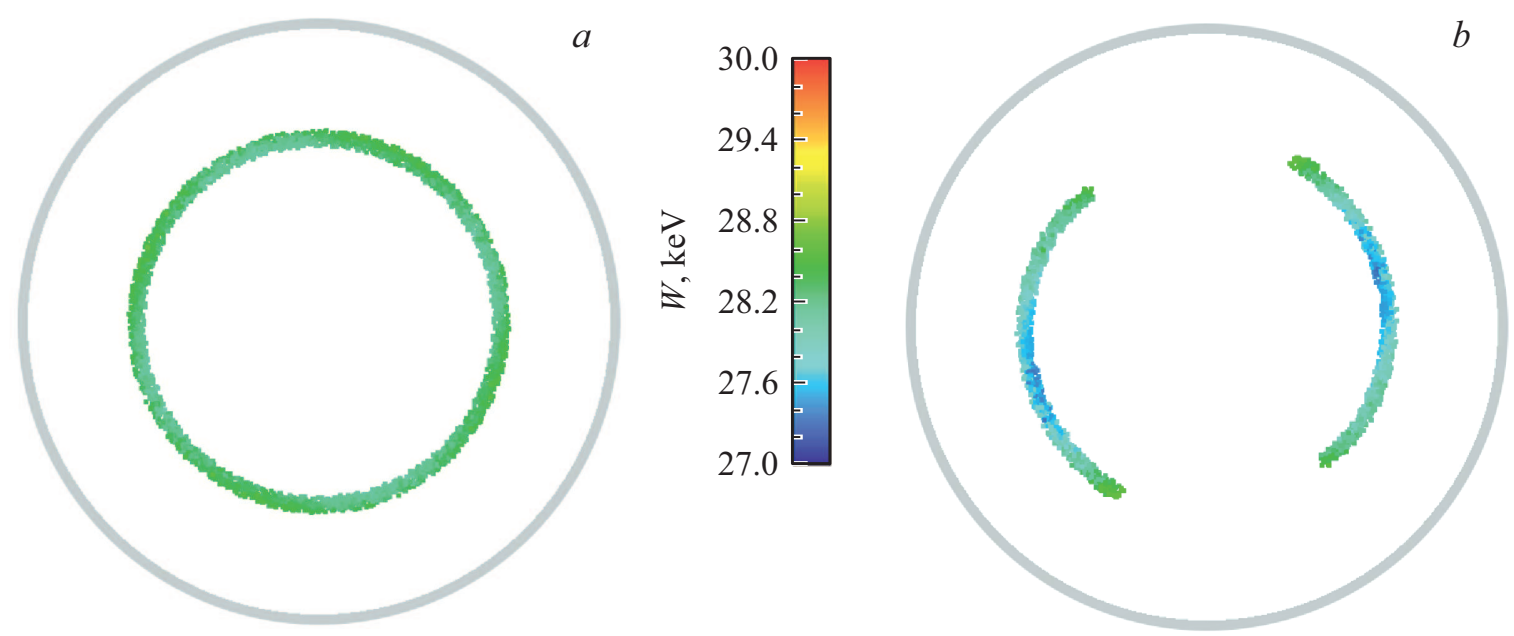

Рис. 2. Распределения частиц в центральной плоскости резонатора $z=260.5 \mathrm{~mm}$, полученные при траекторном анализе ЭОС гиротрона для однородного $(a)$ и секционированного $(b)$ катодов. Цвет частиц соответствует их энергии $W$.

и продольного магнитного полей. Сильное магнитное поле обеспечивает сохранение требуемого распределения электронов вдоль азимутальной координаты для секционированного катода, когда отсутствуют частицы в пределах двух азимутальных секторов.

Важно отметить, в реальных условиях дополнительный скоростной разброс может быть вызван действием факторов, не учтенных в рамках данного моделирования: шероховатостью эмитирующей поверхности катода, неидеальностями изготовления и юстировки элементов лампы, высокочастотными полями, связанными с развитием паразитных неустойчивостей (см., например, [4]). Высокочастотные поля, кроме повышения разброса скоростей, могут также вызывать заметный рост энергетического разброса электронов. При этом шероховатость поверхности катода следует учесть в первую очередь, если рассматривать хорошо спроектированные и отъюстированные ЭОС, в которых подавлено развитие паразитных неустойчивостей. Шероховатость с размером неровностей в единицы микрон, характерная для современных термокатодов, приводит к скоростному разбросу в единицы процентов $[4,25,26]$.

Повышение разброса по скоростям ограничивает возможность увеличения рабочего питч-фактора, поскольку при больших $\alpha$ и $\delta v_{\perp}$ неизбежно отражение электронов от магнитной пробки и развитие паразитных низкочастотных колебаний в ловушке между катодом и резонатором (например, $[4,6,27,28])$. Определенный на основании экспериментальных данных в гиротроне СПбПУ пороговый коэффициент отражения от магнитной пробки 


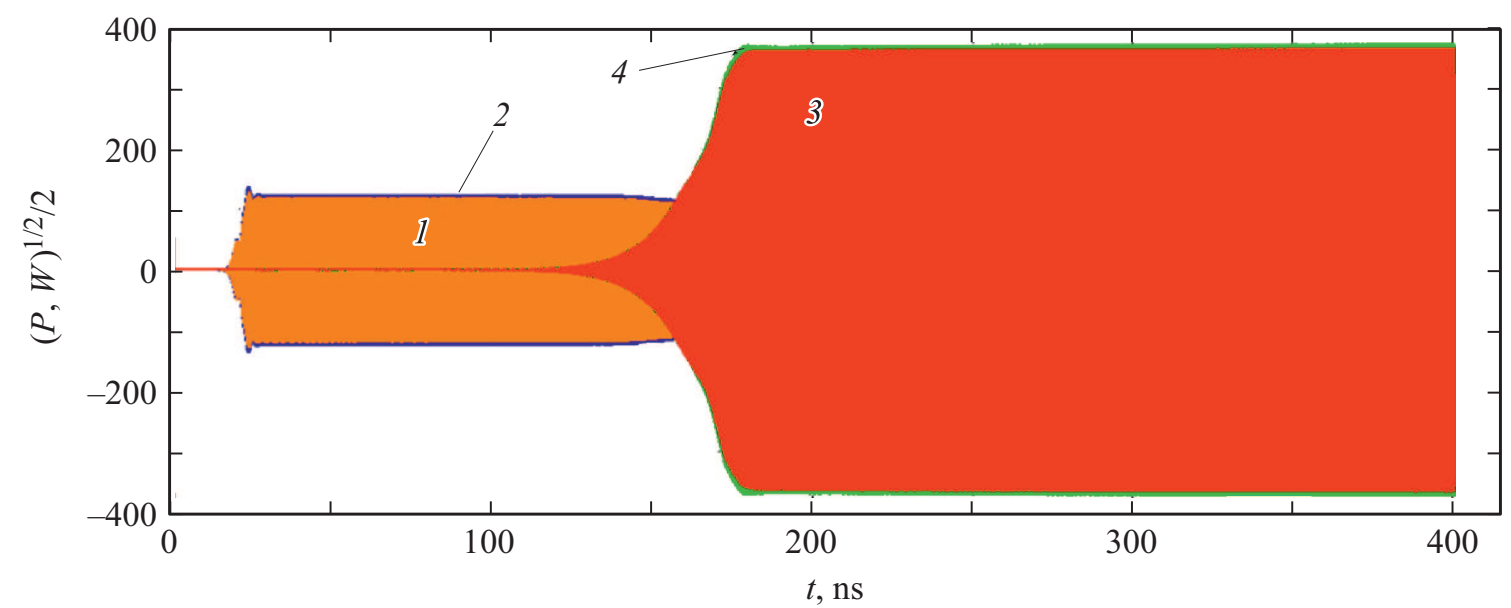

Рис. 3. Изменение во времени сигналов мод $\mathrm{TE}_{11,3}(1,2)$ и $\mathrm{TE}_{12,3}(3,4)$ в выходном порту гиротрона с однородным катодом.

$R_{\mathrm{th}}$, при превышении которого в электронном пространственном заряде существуют данные колебания, составлял примерно $1.7 \cdot 10^{-3}[10]$. При гауссовом распределении по скоростям для среднего питч-фактора $\alpha=1.57$ указанное значение $R_{\text {th }}$ достигается при $\delta v_{\perp}=6.3 \%$, а для $\alpha=1.52-$ при $\delta v_{\perp}=6.7 \%$. В эксперименте максимальный КПД гиротрона $\eta_{\mathrm{el}}=46 \%$ был получен при расчетном питч-факторе $\sim 1.6$ и малом скоростном разбросе, когда не были зафиксированы паразитные низкочастотные колебания [11]. Это дает основание утверждать, что и режимы с питч-фактором $\alpha=1.5-1.6$, исследуемые в настоящей работе, могут быть также реализованы при отсутствии отражения электронов от магнитной пробки.

Данные о параметрах ВЭП, полученные при траекторном анализе ЭОС гиротрона, были использованы на втором этапе при моделировании взаимодействия электронов с высокочастотным полем в резонаторе. Для этого в плоскости $z=222.5 \mathrm{~mm}$ (рис. 1) был установлен специальный монитор Particle Export Interface, который являлся источником частиц в расчетах, описанных в следующем разделе.

\section{2. Моделирование процессов в резонаторе гиротрона}

Проектирование электродинамической системы гиротрона СПбПУ было выполнено ранее сотрудниками ИПФ РАН. Резонатор с радиусом $14.45 \mathrm{~mm}$ и длиной регулярной части $28 \mathrm{~mm}$ (рис. 1) был рассчитан на рабочую моду $\mathrm{TE}_{12,3}$.

Предварительные расчеты позволили определить собственные моды резонатора, а также оптимальные значения среднего радиуса ВЭП и индукции магнитного поля. Моделирование динамических процессов, описанное ниже, было выполнено с помощью вычислителя Particle-inCell (PIC) Solver. Расчетная область в этом случае была ограничена входной плоскостью $z=222.5 \mathrm{~mm}$ и плос- костью $z=320 \mathrm{~mm}$, где был установлен выходной порт, регистрирующий генерируемую СВЧ мощность (рис. 1). Расчетная модель разбивалась примерно на $6 \cdot 10^{6}$ ячеек. Временной шаг интегрирования составлял $\sim 4 \cdot 10^{-4} \mathrm{~ns}$.

При оптимальном значении коэффициента магнитной компрессии $B_{0} / B_{c}=17.01$ средний радиус пучка в центральной плоскости резонатора равняется примерно $8.5 \mathrm{~mm}$. В зоне возбуждения рабочей моды $\mathrm{TE}_{12,3}$ максимальное значение выходной мощности было зафиксировано при индукции магнитного поля в резонаторе $B_{0}=2.747$ T. На рис. 3 показано изменение во времени сигналов мод с наибольшей амплитудой в выходном порту, которые были получены для однородного катода с параметрами ВЭП, указанными в разд. 2, при $B_{0} / B_{c}=17.01$ и $B_{0}=2.747 \mathrm{~T}$. Номера этих мод определены по структуре высокочастотного электрического и магнитного полей. На начальном этапе расчета (примерно до $140 \mathrm{~ns}$ ) генерируется паразитная мода $\mathrm{TE}_{11,3}$ на частоте $~ 71.4 \mathrm{GHz}$. Впоследствии эта мода подавляется одновременно с возбуждением рабочей моды $\mathrm{TE}_{12,3}$. В течение времени $180<t<400 \mathrm{~ns}$ наблюдается стабильная генерация с частотой $\sim 74.5 \mathrm{GHz}$. Для обеих мод имеются две поляризационные составляющие с примерно одинаковой амплитудой (рис. 3), суперпозиция которых обеспечивает круговую поляризацию волны в резонаторе.

В момент времени $t=400 \mathrm{~ns}$ суммарная генерируемая СВЧ мощность (Wave-Particle Power Transfer) $P_{\mathrm{RF}}=141 \mathrm{~kW}$, а электронный КПД $\eta_{\mathrm{el}}=47 \%$ при $U_{0}=30 \mathrm{kV}, I_{b}=10 \mathrm{~A}$. Практически вся мощность сосредоточена в рабочей моде $\mathrm{TE}_{12,3}$ - суммирование сигналов двух составляющих этой моды дает величину $\sim 137 \mathrm{~kW}$. При переходе к секционированному катоду характер возбуждения мод принципиально не меняется. Наблюдается лишь некоторое снижение выходной СВЧ мощности, обусловленное, очевидно, более низким качеством ВЭП. Суммарная выходная мощность для 

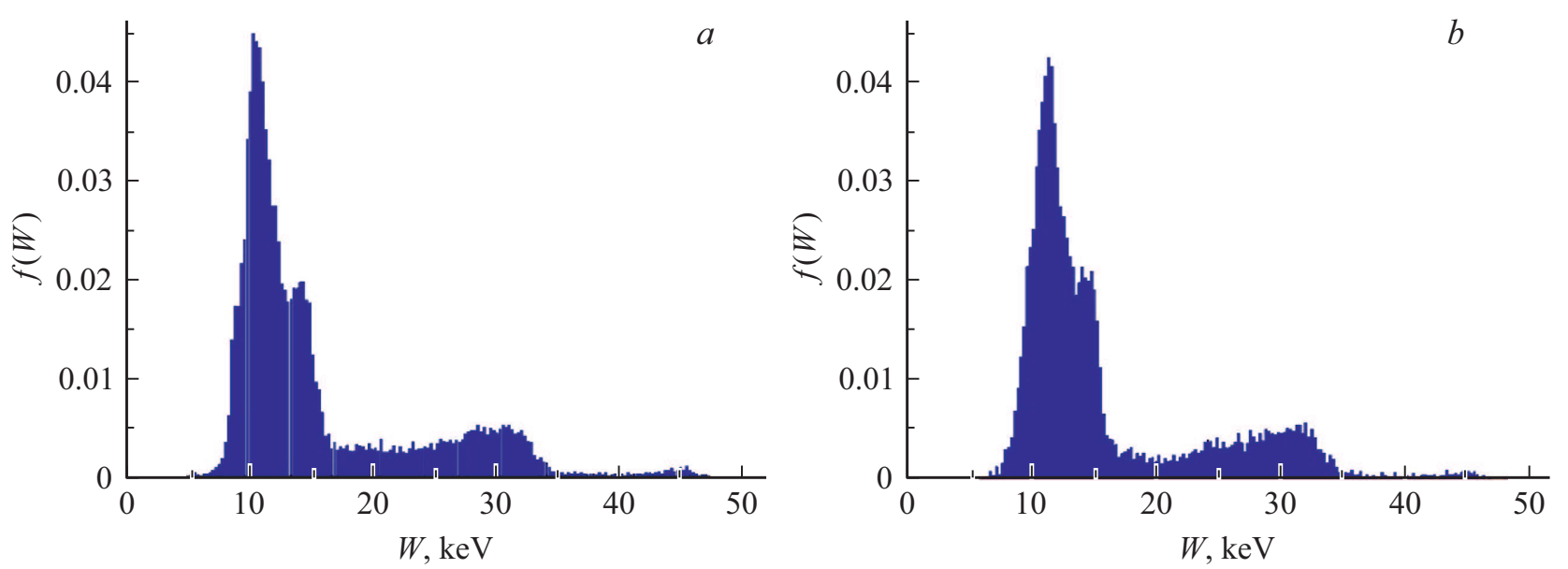

Рис. 4. Гистограммы распределения электронов по энергиям в отработанном ВЭП для однородного $(a)$ и секционированного $(b)$ катодов. Диапазон энергий электронов разбит на 400 интервалов.

этого катода в момент времени $t=400 \mathrm{~ns}$ составляет $\sim 138 \mathrm{~kW}$, а мощность в моде $\mathrm{TE}_{12,3}-128 \mathrm{~kW}$.

Необходимой информацией для проектирования системы рекуперации являются данные о параметрах отработанного ВЭП, прошедшего через резонатор и отдавшего часть своей энергии высокочастотному полю. Эти данные фиксировались в плоскости выходного порта $z=320 \mathrm{~mm}$. Здесь был установлен монитор частиц PIC 2D Monitor, который собирал сведения в течение времени $\Delta t=0.003 \mathrm{~ns}$. При такой величине $\Delta t$ в каждом файле, соответствующем выбранному моменту времени $t$, содержится информация о примерно $25 \cdot 10^{3}$ частиц. На рис. 4 показаны энергетические спектры электронов в отработанном ВЭП для однородного $(a)$ и секционированного $(b)$ катодов. На основании данных о распределении электронов по энергиям были выбраны значения потенциалов секций коллектора, при которых обеспечивается минимум величины мощности, рассеиваемой в виде тепла на этих секциях $[21,22]$.

Используя соотношение

$$
\eta_{\mathrm{el}}=1-\frac{\int_{0}^{\infty} f(W) W d W}{e U_{0}},
$$

по известному энергетическому спектру $f(W)$ можно оценить электронный КПД $\eta_{\mathrm{el}}$. Например, для однородного катода после интегрирования спектра, приведенного на рис. 4, $a$, получаем $\eta_{\mathrm{el}}=47.9 \%$ при полной энергии частиц $e U_{0}=30 \mathrm{keV}$, что довольно близко к значению КПД, определенному по выходной СВЧ мощности.

\section{3. Траекторный анализ отработанного ВЭП в коллекторной области}

В рекуператоре гиротрона СПбПУ используется метод пространственной сепарации электронов, основан- ный на их дрейфе в скрещенных азимутальном магнитном и аксиальном электрическом полях $[19,21,22]$. Модель коллекторной области показана на рис. 5. Азимутальное магнитное поле создается с помощью соленоида с тороидальной намоткой. Катушки Гельмгольца служат для коррекции распределения аксиального магнитного поля вдоль продольной координаты. Выбором геометрии магнитной системы обеспечивается адиабатичность распределения магнитного поля в переходной области между резонатором и коллектором и квазиоднородность этого поля в области рекуперации длиной примерно $350 \mathrm{~mm}$. В этой области амплитуды аксиальной и азимутальной составляющих индукции магнитного поля на радиусе пучка равны примерно 0.04 и $0.08 \mathrm{~T}$.

Электрическое поле создается с помощью четырех секций-электродов конусной формы, потенциалы которых уменьшаются по мере удаления от резонатора. Дрейф в скрещенных электрическом и магнитном полях приводит к пространственной сепарации электронов с разной энергией и их осаждению на секции под различными потенциалами. Корпус коллектора отделен от основного корпуса гиротрона специальным изолятором, что позволяет реализовать схему простой одноступенчатой рекуперации при соединенных друг с другом секциях и корпусе коллектора. В нем также имеются две соединительные трубки, внутри которых расположены „связки“ - проводники, соединяющие витки внутренней обмотки тороидальной катушки с витками ее наружной обмотки (рис. 5). Магнитное поле „связок“ искажает близлежащие траектории электронов и может приводить к их отражению в сторону резонатора. Для снижения негативного влияния „связок“ в данных исследованиях мы использовали секционированный катод с подавленной эмиссией с двух азимутальных секторов эмитирующего пояска. Описанная коллекторная система реализована в экспериментальном гиротроне.

При выполнении траекторного анализа ВЭП в коллекторной области исследовалась возможность достижения 


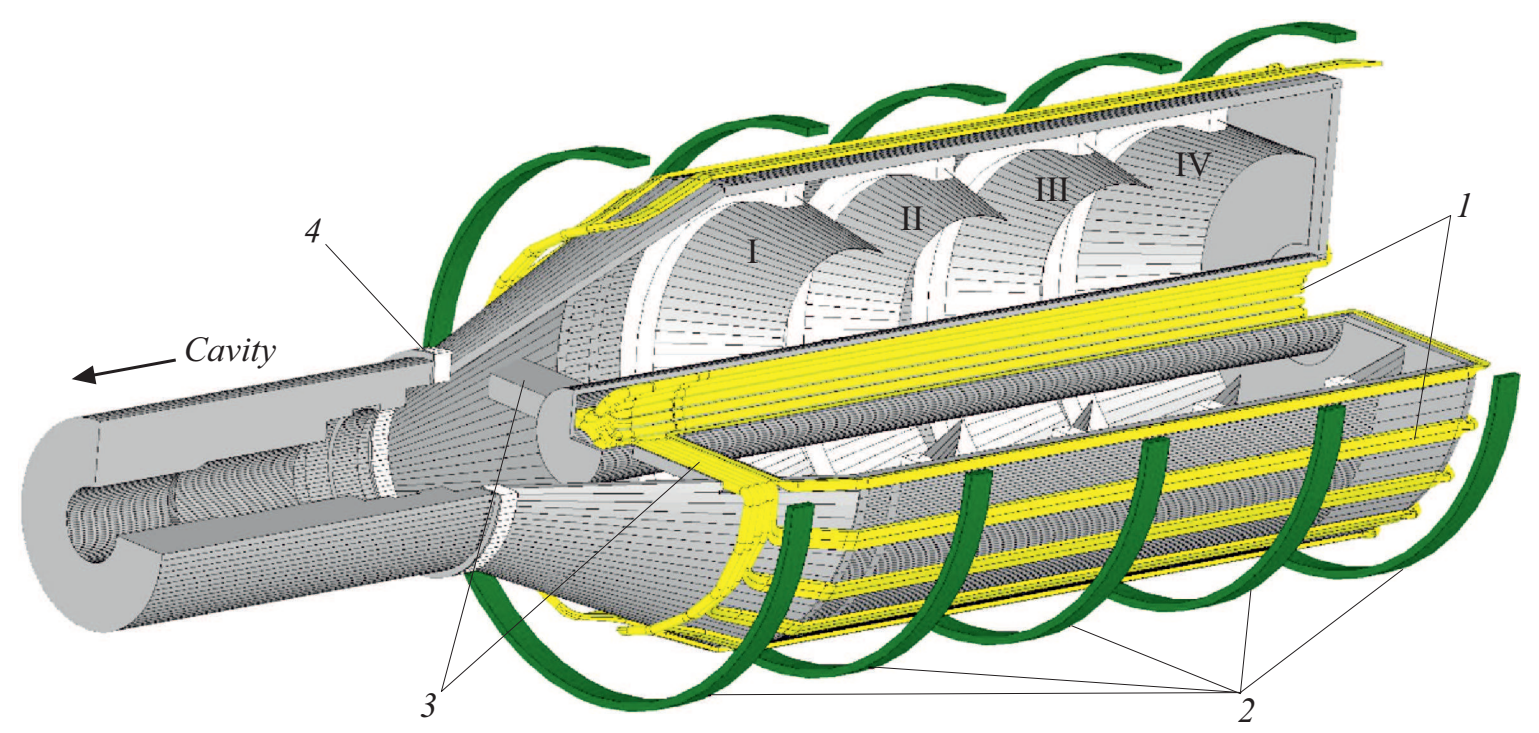

Pис. 5. Схематическое изображение модели коллектора: 1 - соленоид с тороидальной намоткой, 2 - катушки Гельмгольца, 3 „связки“ проводов, 4 - изолятор, I-IV - секции коллектора.

максимальной эффективности рекуперации остаточной энергии, т.е. снижения до минимума величины мощности $P_{\text {diss, }}$ рассеиваемой в виде тепла при осаждении электронов на стенках коллектора. Отметим, что коллектор расположен в области, где индукция магнитного поля заметно меньше величины $B_{0}$ в резонаторе. Поэтому практически вся кинетическая энергия электронов сосредоточена в их продольном (вдоль силовой линии магнитного поля) движении. Минимальная энергия электронов $W_{\min }$ в спектре отработанного ВЭП (рис. 4) определяет величину напряжения $U_{\mathrm{I}}$, подаваемого на первую секцию коллектора. При $\left|e U_{\mathrm{I}}\right|>W_{\min }$ электроны с малыми энергиями могут изменять направление своей продольной скорости, т.е. отражаться в сторону резонатора. Отражение электронов с бо́льшими энергиями может также происходить и в области между секциями. Опасность представляют те электроны, которые после изменения направления скорости не оседают на стенках коллектора, а, выйдя из его области и двигаясь в адиабатически меняющемся магнитном поле, достигают резонатора. Такие электроны, взаимодействуя с высокочастотным полем, могут отбирать от него энергию, что негативно влияет на величину выходной СВЧ мощности. Как показывают проведенные ранее исследования, предельный уровень отражения от коллектора, при превышении которого падает выходная мощность, в различных гиротронах может заметно различаться [5,29-32], и достигать примерно 10\% [33].

В разработанной схеме рекуператора с азимутальным магнитным полем радиальный дрейф приводит к осаждению основной части отраженных электронов на задней стенке секций коллектора, что заметно снижает поток частиц, возвращающихся в резонатор [19]. По этой же причине практически исключено попадание в резонатор вторичных электронов с бомбардируемых поверхностей электродов коллектора.

В настоящей работе решалась многопараметрическая задача по достижению минимального значения мощности $P_{\text {diss }}$ при токе отраженных от коллектора частиц, не превышающем $1-2 \%$ от полного тока ВЭП, за счет оптимизации геометрии и потенциалов секций коллектора, а также азимутального положения секторов катода с подавленной эмиссией. В результате такой оптимизации был получен режим, в котором рассеиваемая на коллекторе мощность $P_{\text {diss }}$ составляла $54.19 \mathrm{~kW}$, а ток электронов, отраженных от коллектора и проходящих в сторону резонатора через входную плоскость $z=320 \mathrm{~mm}$, равнялся $1.37 \%$ от $I_{b}$. В этом режиме корпус коллектора был соединен с корпусом гиротрона, а секции I-IV коллектора имели следующие потенциалы: $U_{\mathrm{I}}=-7.72 \mathrm{kV}, U_{\mathrm{II}}=-10.72 \mathrm{kV}, U_{\mathrm{III}}=-14.72 \mathrm{kV}$, $U_{\mathrm{IV}}=-24.72 \mathrm{kV}$. На рис. 6 показаны $3 D$-траектории электронов в области коллектора. Видно, что использование секционированного эмиттера обеспечивает прохождение электронов в область рекуперации без их осаждения на соединительных трубках, в которых находятся „связки“ проводов тороидальной катушки. По мере движения электронов в тормозящем электрическом поле происходит уменьшение их энергии и смещение на более высокие радиусы под действием скрещенных $E \times B$ полей.

Положение электронных траекторий в плоскости $r-z$ приведены на рис. 7. Выбраны траектории, имеющие разные значения энергии, питч-фактора и радиальной координаты во входной плоскости $z=320 \mathrm{~mm}$ (табл. 2) и проходящие примерно на равном удалении от „связок“. Средний питч-фактор электронов во входной плоскости равен $\sim 0.59$. Поскольку при обмене энергией между ВЭП и высокочастотным полем меняется поперечная 

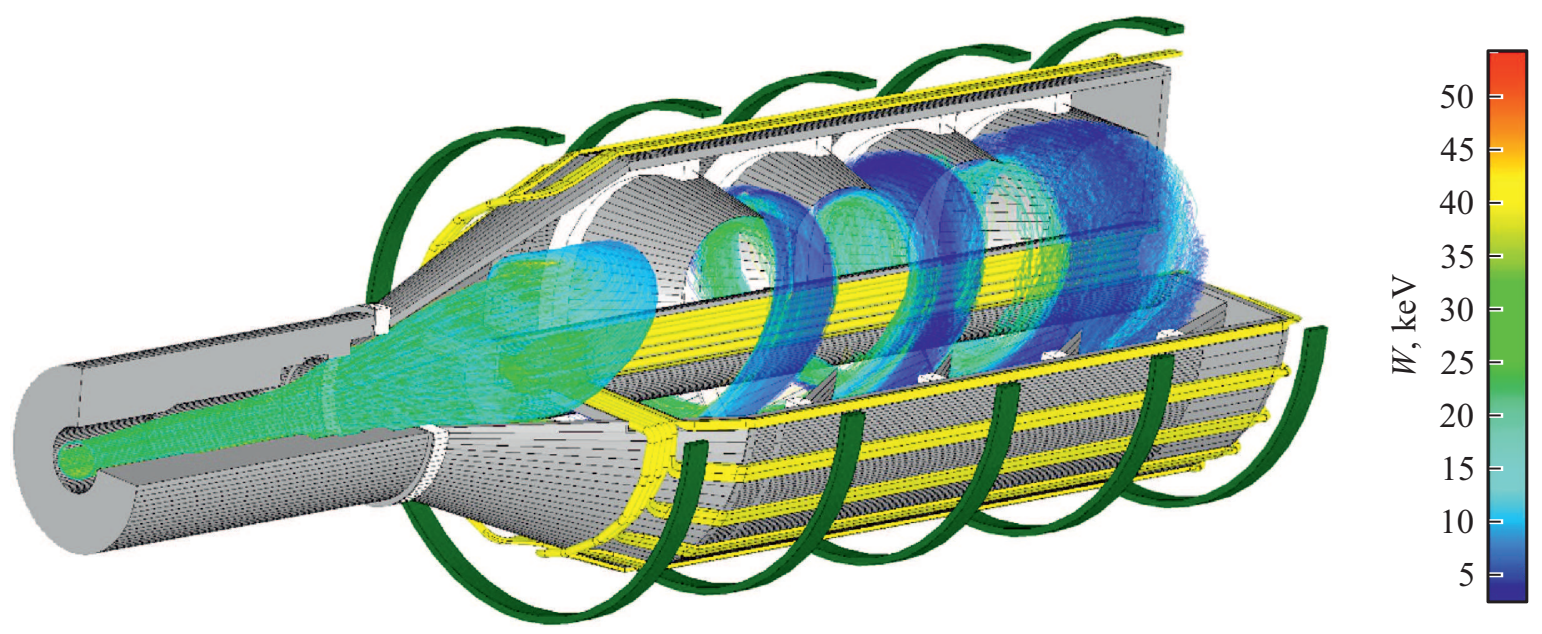

Pис. 6. $3 D$-траектории электронов в коллекторной области. Цвет траекторий соответствует энергии частиц $W$.
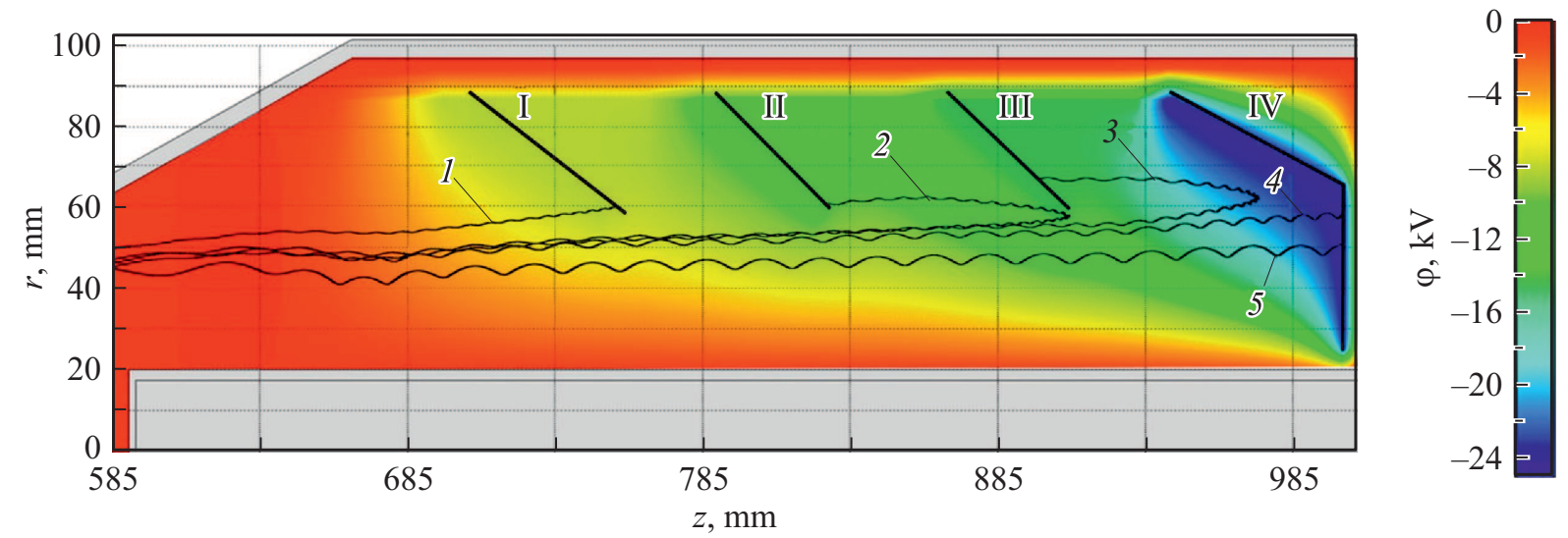

Рис. 7. Траектории электронов $1-5$ и распределение потенциала $\varphi$ в плоскости $r-z$. Элементы магнитной системы и электроды, экранирующие секции I-IV от корпуса коллектора, не показаны.

скорость электронов, то, очевидно, что в среднем с ростом энергии частиц в отработанном пучке возрастает и их питч-фактор. На рисунке показано также распределение потенциала при указанных выше значенияx $U_{\mathrm{I}}-U_{\mathrm{IV}}$. Представленные траектории демонстрируют корректную работу метода пространственной сепарации электронов в скрещенных $E \times B$ полях, обеспечивающего осаждение частиц с разной энергией на секциях под соответствующим потенциалом.

В рассмотренном режиме работы гиротрона его полный КПД

$$
\eta_{t}=\frac{P_{\mathrm{RF}}}{P_{\mathrm{RF}}+P_{\mathrm{diss}}}=71.8 \%,
$$

где $P_{\mathrm{RF}}=138 \mathrm{~kW}, P_{\text {diss }}=54.19 \mathrm{~kW}$. Рассеиваемая на коллекторе мощность распределена по его электродам следующем образом: значения мощности на секциях $P_{\mathrm{I}}=36.15 \mathrm{~kW}, P_{\mathrm{II}}=4.79 \mathrm{~kW}, P_{\mathrm{III}}=5.24 \mathrm{~kW}$, $P_{\mathrm{IV}}=7.58 \mathrm{~kW}$, значение мощности на корпусе коллектора $P_{\text {coll }}=0.43 \mathrm{~kW}$. КПД рекуперации при этом равен $66.5 \%$. В данном рекуператоре основная часть электронов осаждается на первой секции. Это является следствием необходимости уменьшения радиуса внутреннего отверстия этой секции и снижения величины $\left|e U_{\mathrm{I}}\right|$ для того, чтобы обеспечить относительно небольшое отражение электронов в сторону резонатора. Основными причинами отражения электронов и непопадания их на соответствующую секцию являются возмущающее действие магнитного поля „связок“ и имеющийся разброс электронов по радиальным координатам на входе в рекуператор. Если допустимый порог отражения повысить, то можно добиться увеличения полного КПД. Отметим, что для спектра отработанного ВЭП, показанного на рис. $4, b$, в случае 4-х ступенчатого коллектора с идеальной сепарацией, когда любой из электронов попадает на секцию с потенциалом, соответствующим его энергии, максимальный полный КПД равен 81.2\% для остаточной энергии электронов при их осаждении на секциях $W_{\text {rem }}>0.5 \mathrm{keV}$ и $83.6 \%$ для $W_{\text {rem }}>0$ (при отражении от коллектора, равном $1.37 \%$ ).

Возможности дальнейшего повышения эффективности разработанного метода рекуперации связаны, очевидно, с улучшением конструкции магнитной системы, обес- 
Таблица 2. Входные параметры электронных траекторий

\begin{tabular}{c|c|c|c}
\hline № & Энергия, keV & Питч-фактор & Радиус, $\mathrm{mm}$ \\
\hline 1 & 8.270 & 0.45 & 9.34 \\
2 & 13.781 & 0.45 & 8.7 \\
3 & 21.264 & 0.74 & 9.04 \\
4 & 28.097 & 0.83 & 9.03 \\
5 & 46.368 & 1.65 & 8.63
\end{tabular}

печивающей требуемое распределение азимутального магнитного поля. Авторы продолжают поиск оптимального способа создания такого поля, что позволило бы увеличить полный КПД гиротронов, включая мощные гиротроны, работающие в длинноимпульсном и квазинепрерывном режимах.

\section{Заключение}

Таким образом, выполненное в работе комплексное моделирование показало возможность достижения рекордных значений полного КПД импульсного гиротрона средней мощности 4-mm диапазона длин волн, полученное в результате повышения качества ВЭП в электронно-оптической системе и рекуперации остаточной энергии электронов в многоступенчатой коллекторной системе. Разработана конструкция 4-х ступенчатого рекуператора, основанного на методе пространственной сепарации электронов в скрещенных азимутальном магнитном и аксиальном электрическом полях. Использование секционированного катода с подавленной эмиссией с двух азимутальных секторов позволяет повысить эффективность рекуперации и уменьшить коэффициент отражения электронов от коллектора. В оптимальном режиме работы гиротрона был достигнут полный КПД $71.8 \%$ при КПД рекуперации $66.5 \%$ и токе отраженных от коллектора электронов, составляющем $1.37 \%$ от полного тока ВЭП.

\section{Финансирование работы}

Исследование выполнено за счет гранта Российского научного фонда (проект № 16-12-10010). Часть результатов была получена с использованием вычислительных ресурсов суперкомпьютерного центра Санкт-Петербургского политехнического университета Петра Великого (http://www.scc.spbstu.ru).

\section{Конфликт интересов}

Авторы заявляют, что у них нет конфликта интересов.

\section{Список литературы}

[1] Litvak A.G., Denisov G.G., Myasnikov V.E., Tai E.M., Azizov E.A, Ilin V.I. // J. Infrared Millimeter Terahertz Waves. 2011. Vol. 32. N 3. P. 337-342.

[2] Thumm M. State-of-the-art of high power gyro-devices and free electron masers, update 2017. KIT Scientific Report 7750. Karlsruhe Institute of Technology, 2018. 183 p.

[3] Запевалов В.Е. // Известия вузов. Радиофизика. 2006. Т. 49. Вып. 10. C. 864-871. [Zapevalov V.E. // Radiophys. Quantum Electron. 2006. Vol. 49. N 10. P. 779-785.]

[4] Tsimring Sh.E. // Int. J. Infrared Millimeter Waves. 2001. Vol. 22. N. 10. P. 1433-1468.

[5] Завольский Н.А., Запевалов В.Е., Куфтин А.Н., Постникова A.C. // Материалы XXVIII междунар. крымской конф. „СВЧ-техника и телекоммуникационные технологии“, Севастополь, 2018. С. 1131-1137.

[6] Лукша О.И. Винтовые электронные потоки гиротронов: динамика пространственного заряда и методы повышения качества. Докт. дис. СПб.: С.-Петербург, СанктПетербургский государственный политехнический университет (СПбГПУ), 2011. 285 с.

[7] Касьяненко Д.В., Лукша О.И., Пиосчик Б., Соминский Г.Г., Тумм М. // Известия вузов. Радиофизика. 2004. Т. 47. Вып. 5-6. C. 463-470. [Kas'yanenko D.V., Louksha O.I., Piosczyk B., Sominsky G.G., Thumm M. // Radiophys. Quantum Electron. 2004. Vol. 47. N 5-6. P. 414-420.]

[8] Louksha O., Piosczyk B., Sominski G., Thumm M., Samsonov D. // IEEE Trans. Plasma Sci. 2006. Vol. 34. N. 3. P. 502-511.

[9] Лукша О.И., Самсонов Д.Б., Соминский Г.Г., Цапов А.А. // ЖТФ. 2012. Т. 82. Вып. 6. С. 101-105. [Louksha O.I., Samsonov D.B., Sominskii G.G., Tsapov A.A. // Tech. Phys. 2012. Vol. 57. N 6. P. 835-839.]

[10] Лукша О.И., Самсонов Д.Б., Соминский Г.Г., Семин С.В. // ЖТФ. 2013. Т. 83. Вып. 5. С. 132-140. [Louksha O.I., Samsonov D.B., Sominskii G.G., Semin S.V. // Tech. Phys. 2013. Vol. 58. N 5. P. 751-759.]

[11] Louksha O.I., Sominski G.G., Arkhipov A.V., Dvoretskaya N.V., Kolmakova N.G., Samsonov D.B., Trofimov P.A. // IEEE Trans. Plasma Sci. 2016. Vol. 44. N 8. P. $1310-1319$.

[12] Manuilov V.N., Morozkin M.V., Luksha O.I., Glyavin M.Y. // Infrared Physics and Tech- nology. 2018. Vol. 91. N P. 46-54.

[13] Kosmahl H.G. // Proc. IEEE. 1982. Vol. 70. N 11. P. 13251334.

[14] Goldenberg A.L., Manuilov V.N., Moiseev M.A., Zavolsky N.A. // Int. J. Infrared Millimeter Waves. 1996. Vol. 18. N 1. P. 43-55.

[15] Singh A., Rajapatirana S., Men Y., Granatstein V.L., Ives R.L., Antolak A.J. // IEEE Trans. Plasma Sci. 1999. Vol. 27. N. 2. P. $490-502$.

[16] Ling G., Piosczyk B., Thumm M.K. // IEEE Trans. Plasma Sci. 2000. Vol. 28. N 3. P. 606-613.

[17] Глявин М.Ю., Морозкин М.В., Петелин М.И. // Известия вузов. Радиофизика. 2006. Т. 49. Вып. 10. С. 900-905. [Glyavin M.Y., Morozkin M.V., Petelin M.I. // Radiophys. Quantum Electron. 2006. Vol. 49. N 10. P. 811-815.]

[18] Pagonakis I.Gr., Hogge J.-P., Alberti S., Avramides K.A., Vomvoridis J.L. // IEEE Trans. Plasma Sci. 2008. Vol. 36. N 2. P. 469-480.

[19] Лукша О.И., Тробимов П.А. // Письма в ЖТФ. 2015. Т. 41. Вып. 18. C. 38-45. [Louksha O.I., Trofimov P.A. // Tech. Phys. Lett. 2015. Vol. 41. N 9. P. 884-886.] 
[20] Wu C., Pagonakis I.G., Avramidis K.A. Gantenbein G., Illy S., Thumm M., Jelonnek J. // Phys. Plasmas. 2018. Vol. 25. N. 3. Article number 033108.

[21] Louksha O.I., Trofimov P.A. // Proc. 41st Int. Conf. Infrared, Millimeter, and Terahertz Waves, Copenhagen, Denmark, 2016, article number 7758519.

[22] Louksha O.I., Trofimov P.A. // Proc. 18th Int. Vacuum Electronics Conf., IVEC 2017, London, United Kingdom, 2017. P. 1-2.

[23] Электронный ресурс. Режим доступа: http://www.cst.com

[24] Louksha O.I., Trofimov P.A. // ЖТФ. 2018. Т. 88. Вып. 4. C. 614-620. [Louksha O.I., Trofimov P.A. // Tech. Phys. 2018. Vol. 63. N 4. P. 598-604.

[25] Авдошин Е.Г., Николаев Л.В., Платонов И.Н., Цимринг Ш.Е. // Известия вузов. Радиофизика. 1973. Т. 16. Вып. 4. С. 605-612. [Avdoshin E.G., Nikolaev L.V., Platonov I.N., Tsimring Sh.E. // Radiophys. Quantum Electron. 1973. Vol. 16. N 4. P. 461-466.]

[26] Zhang J., Illy S., Pagonakis I., Avramidis K.A., Thumm M., Jelonnek J. // Nuclear Fusion. 2015. Vol. 56. N 2. Article number 026002.

[27] Мануилов В.Н. // Известия вузов. Радиофизика. 2006. Т. 49. Вып. 10. C. 872-879. [Manuilov V.N. // Radiophys. Quantum Electron. 2006. Vol. 49. N 10. P. 786-792.

[28] Лукша О.И. // Известия вузов. Радиофизика. 2009. Т. 52. Вып. 5-6. C. 425-437. [Luksha O.I. // Radiophys. Quantum Electron. 2009. Vol. 52. N 5-6. P. 386-397.]

[29] Saraph G.P., Felch K.L., Feinstein J., Borchard P., Cauffman S.R., Chu S. // IEEE Trans. Plasma Sci. 2000. Vol. 28. N 3. P. $830-840$.

[30] Sakamoto K., Tsuneoka M., Kasugai A., Imai T., Kariya T., Hayashi K., Mitsunaka Y. // Phys. Rev. Lett. 1994. Vol. 73. N 26. P. 3532-3535.

[31] Венедиктов Н.П., Глявин М.Ю., Запевалов В.Е., Кубтин А.Н. // Известия вузов. Радиофизика. 1998. Т. 41. Вып. 5. С. 670-678. [Venediktov N.P., Glyavin M.Yu., Zapevalov V.E., Kuftin A.N. // Radiophys. Quantum Electron. 1998. Vol. 41. N 5. P. 449-456.].

[32] Morozkin M.V., Glyavin M.Y., Denisov G.G., Luchinin A.G. // Int. J. Infrared Millimeter Waves. 2008. Vol. 29. N 11. P. 1004-1010.

[33] Piosczyk B., Iatrou C.T., Dammertz G., Thumm M. // IEEE Trans. Plasma Sci. 1996. Vol. 24. N 3. P. 579-585. 